\title{
Genetic Diversity of Hard Ticks (Acari: Ixodidae) in the South and East Regions of Kazakhstan and Northwestern China
}

\begin{abstract}
Yicheng Yang ${ }^{1,2, \dagger}\left(\mathbb{0}\right.$, Jin Tong ${ }^{3, \dagger}$, Hongyin Ruan ${ }^{4, \dagger}$, Meihua Yang ${ }^{5}$, Chunli Sang', Gang Liu', Wurelihazi Hazihan ${ }^{6}$, Bin $\mathrm{Xu}^{7}$, Sándor Hornok ${ }^{8}$, Kadyken Rizabek ${ }^{9}$, Kulmanova Gulzhan ${ }^{9}$, Zhiqiang Liu ${ }^{10}$, Yuanzhi Wang ${ }^{1, *}$ (i)

${ }^{1}$ Department of Basic Medicine, School of Medicine, Shihezi University, Shihezi City, Xinjiang Uygur Autonomous Region 832002, People's Republic of China; 'Emergency Department, Shihezi City People's Hospital, Shihezi City, Xinjiang Uygur Autonomous Region 832000, People's Republic of China; ${ }^{3}$ Department of Ultrasound, the First Affiliated Hospital of Medical College, Shihezi University, Shihezi City, Xinjiang Uygur Autonomous Region 832008, People's Republic of China; ${ }^{4}$ Department of Stomatology, School of Medicine, Shihezi University, Shihezi City, Xinjiang Uygur Autonomous Region 832002, People's Republic of China; ${ }^{5}$ Department of Forestry, College of Agriculture, Shihezi University, Shihezi City, Xinjiang Uygur Autonomous Region 832002, People's Republic of China; ${ }^{6}$ School of Animal Science and Technology, Shihezi University, Shihezi City, Xinjiang Uygur Autonomous Region 832002, People's Republic of China; ${ }^{7}$ National Institute of Parasitic Diseases, Chinese Center for Disease Control and Prevention, WHO Collaborating Centre for Tropical Diseases, Key Laboratory of Parasitology and Vector Biology of the Chinese Ministry of Health, Shanghai 200025, China; ${ }^{8}$ Department of Parasitology and Zoology, University of Veterinary Medicine, Budapest 1078, Hungary; ${ }^{9}$ Department of Food Engineering, Kazakh National Agrarian University, Almaty Oblast 050010, Kazakhstan; ${ }^{10}$ Institute of Veterinary Medicine, Xinjiang Academy of Animal Science, Urumqi City, Xinjiang Uygur Autonomous Region 830000, People's Republic of China
\end{abstract}

\begin{abstract}
To date, there is no report on the genetic diversity of ticks in these regions. A total of 370 representative ticks from the south and east regions of Kazakhstan (SERK) and Xinjiang Uygur Autonomous Region (XUAR) were selected for molecular comparison. A fragment of the mitochondrial cytochrome $c$ oxidase subunit I (cox1) gene, ranging from $631 \mathrm{bp}$ to $889 \mathrm{bp}$, was used to analyze genetic diversity among these ticks. Phylogenetic analyses indicated 7 tick species including Hyalomma asiaticum, Hyalomma detritum, Hyalomma anatolicum, Dermacentor marginatus, Rhipicephalus sanguineus, Rhipicephalus turanicus and Haemaphysalis erinacei from the SERK clustered together with conspecific ticks from the XUAR. The network diagram of haplotypes showed that i) Hy. asiaticum from Almaty and Kyzylorda Oblasts together with that from Yuli County of XUAR constituted haplogroup H-2, and the lineage from Chimkent City of South Kazakhstan was newly evolved; and ii) the $R$. turanicus ticks sampled in Israel, Almaty, South Kazakhstan, Usu City, Ulugqat and Baicheng Counties of XUAR were derivated from an old lineage in Alataw City of XUAR. These findings indicate that: i) Hy. asiaticum, R. turanicus and Ha. erinacei shared genetic similarities between the SERK and XUAR; and ii) Hy. marginatum and $D$. reticulatus show differences in their evolution.
\end{abstract}

Key words: Genetic diversity, hard tick, Kazakhstan, northwestern China

The Republic of Kazakhstan is located in Central Asia between $39^{\circ} 49^{\circ}-55^{\circ} 49^{\circ} \mathrm{N}$ and $46^{\circ} 28^{\circ}-87^{\circ} 18^{\circ} \mathrm{E}$, with its western part extending into Eastern Europe. Kazakhstan is the ninth largest country in the world with a total area of 2,727,300 $\mathrm{km}^{2}$, also ranking as the world's largest landlocked country $[1,2]$. It borders Russia, Kyrgyzstan, Turkmenistan, Uzbekistan and China [3]. Xinjiang Uygur Autonomous Region (XUAR), occupies $1 / 6$ of China and borders 8 countries, covering an

- Received 25 August 2020, revised 17 January 2021, accepted 19 January 2021.

*Corresponding author (wangyuanzhi621@126.com)

†These authors contributed equally to this work.

(c) 2021, Korean Society for Parasitology and Tropical Medicine

This is an Open Access article distributed under the terms of the Creative Commons Attribution Non-Commercial License (https://creativecommons.org/licenses/by-nc/4.0) which permits unrestricted non-commercial use, distribution, and reproduction in any medium, provided the original work is properly cited. area of approximately 1,660,000 $\mathrm{km}^{2}$ [4]. The borderline between the south and east regions of Kazakhstan (SERK) and XUAR is $1,783 \mathrm{~km}$ long [5]. The ecological environment, topography, climate and natural landscape are similar both in the SERK and XUAR [6].

The mitochondrial cytochrome $c$ oxidase subunit I ( $\operatorname{cox} 1)$ gene, also regarded as DNA barcoding, was informative in resolving recent diverging events between closely related species and at the intraspecific level [7]. Recently, 14 tick species, including Dermacentor nuttalli, Dermacentor niveus, Dermacentor marginatus, Dermacentor silvaru, Hyalomma asiaticum, Hyalomma detritum, Haemaphysalis punctata, Haemaphysalis concinna, Haemaphysalis erinacei, Rhipicephalus turanicus, Rhipicephalus sanguineus, Argas vespertilionis, Argas persicus, and Ixodes kaiseri, 
have been molecularly characterized in the border regions of XUAR [8]. At the same time, while at least 13 tick species have been identified in Kazakhstan, including Dermacentor daghestanicus, D. marginatus, D. niveus, Dermacentor reticulatus, Hy. asiaticum, Hyalomma anatolicum, Hyalomma scupense, Hyalomma marginatum, Haemaphysalis punctata, Haemaphysalis sulcata, Rhipicephalus turanicus, Boophilus calcaratus and Ixodes persulcatus $[9,10]$, and their molecular characteristics are still unknown. Here we report the genetic diversity of ticks in the SERK, in comparison with ticks from the XUAR.

During 2015-2019, a total of 4,392 hard ticks were collected from 605 domestic animals (287 sheep, 210 cattle, 101 camels, 7 dogs) and 16 hedgehogs at 24 sampling sites belonging to 15 districts of 5 Oblasts (East Kazakhstan, Almaty, Jambyl, South Kazakhstan and Kyzylorda) in the SERK. After morphological identification, 213 representative ticks were selected for further molecular analysis. Meanwhile, 157 representative ticks collected from marbled polecats, sheep and camels between 2015 and 2018 in 8 districts of the XUAR were used for comparison.

The genomic DNA was extracted from the selected 370 ticks individually using TIANamp Genomic DNA Kit (TIANGEN, Beijing, China) according to the manufacturer's instructions. In this study, we methodologically omitted shorter than 450 bp cox 1 sequences, and chose 631-889 bp fragment to analyze inter- and intra-species genetic diversity. The primers used for PCR were LCO1490 (5'-GGTCAACAAATCATAAAGATATTGG-3') and HCO2198 (5'-TAAACTTCAGGGTGACCAAAAAATCA-3') [11]. The cycling conditions consisted of an initial 5-min denaturation at $95^{\circ} \mathrm{C}$, followed by 37 cycles at $94^{\circ} \mathrm{C}$ for $40 \mathrm{sec}, 55^{\circ} \mathrm{C}$ for $40 \mathrm{sec}$, and $72^{\circ} \mathrm{C}$ for $1 \mathrm{~min}$, with a final extension at $72^{\circ} \mathrm{C}$ for $10 \mathrm{~min}$.

Sequences of the cox 1 were compared with GenBank data using BLAST program (http://www.ncbi.nlm.nih.gov/BLAST/) after manual editing. This dataset was resampled 1,000 times to generate bootstrap values. Phylogenetic relationships were inferred using the Maximum Likelihood (ML) method. The best-fitting substitution model was determined with the Akaike Information Criterion using the ML model test implemented in MEGA 7.0 software [12]. Genetic diversity was estimated as haplotype (h) and nucleotide diversity $(\pi)$ indices with the programme DNAsp v5.10.1 [13]. Median-joining (MJ) networks were generated with the software Network v5.10.1 [14] to display the configuration of haplotypes [15].

Morphological and molecular identification revealed the presence of 9 tick species in the 5 evaluated southeastern border oblasts of Kazakhstan, including Hy. asiaticum, Hy. detritum, Hy. anatolicum, Hy. marginatum, D. marginatus, D. reticulatus, $R$. sanguineus, $R$. turanicus and Ha. erinacei. Sequence identities ranged from $96.27 \%$ to $100 \%$ using BLAST analysis of the cox 1 in intra-species. The sequences in this study were deposited in GenBank (Accession No.: MF002579-MF002581, MK213071, MK307807, MK610453, MN817302, MN841460MN841462, MN841464, MN853165-MN853167, MN862754, MN865123, MN868560, MN868592, MN892553, MN961479, MT506455, MT664833, KU364300-KU364301, KU364303, KU364307, KX882100, KY996841).

Phylogenetic analysis based on the cox 1 (Fig. 1) revealed that: i) Hy. detritum ticks from East Kazakhstan Oblast (MN841460) clustered together with conspecific ticks from Turpan Region of XUAR (KF583581) and Inner Mongolia Province of China (JQ737068); ii) Ha. erinacei ticks from Almaty Oblast, Kazakhstan (MN841464), and Altaw City of XUAR (KU364301) belonged to a different phylogenetic group than ticks from European countries; iii) Hy. anatolicum ticks from Jambyl Oblast (MN853167), Karshgar City of XUAR (KF583576) and Iran (KT920180) represented an ancestral clade to ticks from Iraq, India and Pakistan; iv) D. marginatus ticks from Qapqal County of XUAR (JX051151), East Kazakhstan Oblast (MN868592), and South Kazakhstan Oblast (MN868560) showed a separated branch with ticks from D. marginatus in Alataw City of XUAR (KU364300) with high (100\%) bootstrap support; and v) R. sanguineus sensu lato from Alamaty Oblast (MN862754) was more closely related to a specimen from Iran than to ticks from Altaw City of XUAR (KU364307).

Haplotypes is defined as specific combinations of alleles on an individual chromosome [16]. The network diagram of $H y$. asiaticum showed the haplotypes in the SERK and XUAR based on the cox1. The analyses are as follows: i) the H-2 haplotype was the most dominant and the widespread in SERK and XUAR, such as Almaty Oblast (MN892553), Kyzylorda Oblast (MN961479) and Yuli County of XUAR (KF527400); ii) the $\mathrm{H}-5, \mathrm{H}-6$, and $\mathrm{H}-7$ haplotypes were originated from the H-2 haplotype; and iii) the H-3 and $\mathrm{H}-4$ haplotypes were furtherly originated from the H-6 haplotype. Interestingly, the H-1 haplotype, being distributed in South Kazakhstan, was derivated from the H-3 haplotype, which was distributed in Shihezi City, Fuhai County and Alataw City of XUAR. Meanwhile, 3 lineages of $R$. turanicus from Almaty and South Kazakhstan Oblast 


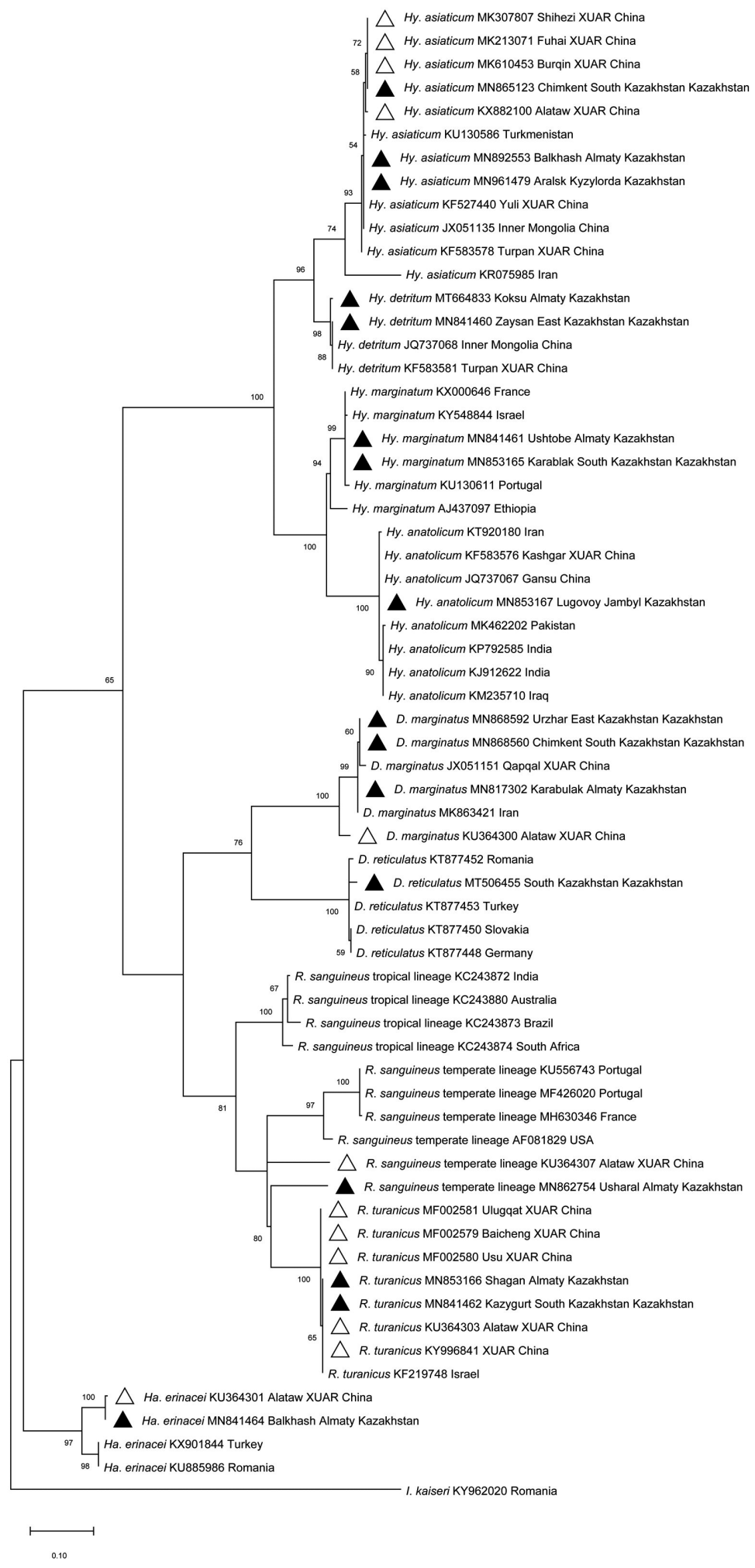

Fig. 1. Maximum-likelihood (ML; 1,000 bootstrap replicates) phylogenetic tree of the cox1 constructed with MEGA 7.0 software, using the sequences of tick species from $\operatorname{SERK}(\boldsymbol{\Delta})$ and $\operatorname{XUAR}(\triangle)$ in this study and the sequences available in the GenBank. The scale bar represented the inferred substitutions per nucleotide site. 
(MN853166 and MN841462, H-1 haplotype), XUAR (MF002579MF002581, H-3 haplotype), and Israel (KF219748, H-4 haplotype), originated from an ancestral lineage from Alataw City of XUAR (KU364303, H-2 haplotype) (Fig. 2). In addition, our study revealed many highly divergent haplotypes for $\mathrm{H}$. asiaticum, e.g. H-1, H-4, H-7, and H-8, while many highly divergent haplotypes for $R$. turanicus, e.g. $\mathrm{H}-1, \mathrm{H}-3$, and $\mathrm{H}-4$, this phenomenon maybe be related to a long and complex evolutionary history of Hy. asiaticum and R. turanicus in the XUAR and SERK.

Based on ecological feature, Hy. scupense is a one-host tick while Hy. detritum is a 2-host species, although they are identical in morphological characteristics [17]. Here Hy. detritum ticks were all sampled from herbivorous livestock, and phylo- genetic analyses showed that Hy. detritum in East Kazakhstan Oblast (MN841460) shared a common branch with these in Turpan Region (the north of XUAR, KF583581) and Inner Mongolia Province of China (JQ737068), and that Hy. detritum ticks in Almaty Oblast (MT664833) were their ancestral lineages.

The brown dog tick R. sanguineus (Latreille, 1806), which is generally accepted as the taxonomic baseline for the R. sanguineus group, is the most widely distributed tick species globally [18]. At least 2 lineages of $R$. sanguineus seno lato (s.l), namely temperate and tropical lineages, were presented [19]. Here phylogenetic analysis indicated $R$. sanguineus both Almaty Oblast in Kazakhstan (MN862754) and Alataw City of XUAR (KU364307) belonged to R. sanguineus s.l temperate

\section{A network diagram of Hyalomma asiaticum in the haplotypes}

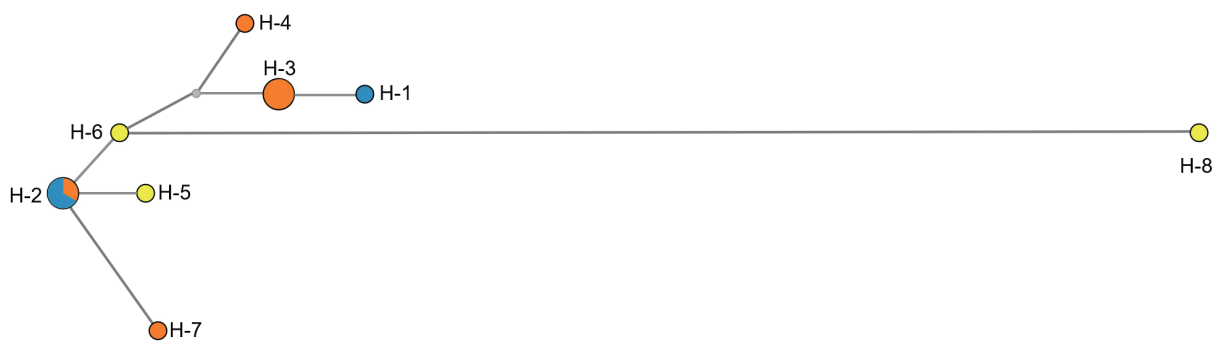

H-1: MN865123

$\mathrm{H}-2$ : MN892553, MN961479, KF527440

H-3: MK610453, MK307807, MK213071

H-4: KX882100

H-5: KU130586

H-6: JX051135

$\mathrm{H}-7$ : KF583578

H-8: KR075985

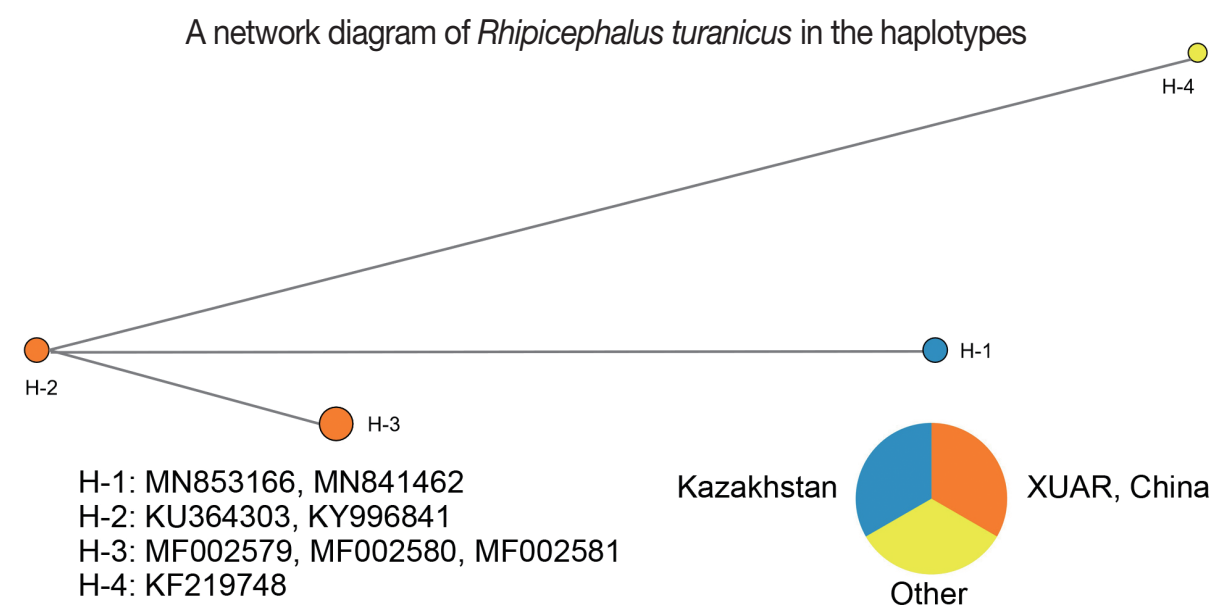

Fig. 2. Network diagrams of Hyalomma asiaticum and Rhipicephalus turanicus showing the haplotypes in the SERK and XUAR based on the mitochondrial cox1 gene. 
lineages, which was a separated clade with $R$. sanguineus tropical lineage from India, Australia, Brazil and South Africa.

The mitochondrial cox1, as the standard DNA barcode, is the best choice for identification of tick species [7]. In the present study, 7 tick species from the SERK, including $H y$. asiaticum, Hy. detritum, Hy. anatolicum, D. marginatus, $R$. sanguineus, R. turanicus and Ha. erinacei, were clustered together with conspecific ticks from the XUAR. Nevertheless, the mitochondrial lineages were unknown in $H y$. marginatus and D. reticulatus ticks from the SERK and XUAR, as for the cox 1 data of Hy. marginatum and D. reticulatus from the XUAR were absent. Animal transportation likely contributed to the dispersal of different haplotypes. Movements of wildlife and migratory birds are well-documented in these regions [20], which might contribute to common genetic lineages of tick species. In the future, it is vital to sample more tick species both in the SERK and XUAR, which is helpful to explore their commonness and difference in tick's evolution.

\section{ACKNOWLEDGMENTS}

This research was supported in part by the National Key Research \& Development Program of China (2018ZX10101002007 and 2018ZX10101002-003), National Natural Science Foundation of China (81960379 and 31960709), Humanities and Social Sciences Research Projects (18YJCZH220), Highlevel Talent Project of Shihezi University (RCZK2018C04), and the International Cooperation Projects of Xinjiang Uygur Autonomous Region (2020E01008).

\section{CONFLICT OF INTEREST}

The authors declare that they have no conflict of interest.

\section{REFERENCES}

1. Cherednichenko A. Estimating agricultural adaptation to climate change through cloud activation for northern Kazakhstan. In Qi J, Evered KT eds, Environmental Problems of Central Asia and Their Economic, Social and Security Impacts. Tashkent, Uzbekistan. Springer. 2008, pp. 183-190. https://doi.org/10.1007/9781-4020-8960-2

2. Yamanaka H, Murata K, Tabata R, Kawaguchi F, Sasazaki S, Yamamoto Y, Bakhtin M, Kazymbet P, Meldevekob A, Suleimenov MZ, Nishibori M, Mannen H. Kazakhstani Native Cattle Reveal Highly Divergent mtDNA From Bos Taurus and Bos Indicus Lin- eages With an Absence of Bos Indicus Y Chromosome. Anim Sci J 2019; 90: 29-34. https://doi.org/10.1111/asj.13128

3. Bismil'din FB, Shapieva ZZ, Anpilova EN. Current malaria situation in the Republic of Kazakhstan. Med Parazitol (Mosk) 2001; 181: 24-33 (in Russian).

4. Zhou M, Cao S, Sevinc, F, Sevinc M, Ceylan O, Moumouni PF, Jirapattharasate C, Liu M, Wang G, Iguchi A, Vudriko P, Suzuki $\mathrm{H}$, Xuan X. Molecular detection and genetic identification of $\mathrm{Ba}$ besia bigemina, Theileria annulata, Theileria orientalis and Anaplasma marginale in Turkey. Ticks Tick Borne Dis 2016; 7: 126-134. https://doi.org/10.1016/j.ttbdis.2015.09.008

5. Mihailovich SS, Isaevna MG. Land border delimitation of Republic of Kazakhstan: practical knowledge of border policy formation. Eur Sci Rev 2018; 2018: 202-204. https://doi.org/10.29013/ESR18-7.8-202-204

6. Hanapia S, Peng D, Junqi Y, Li C. Species diversity characteristics and conservation status in Xinjiang and Kazakhstan, China. Anhui Agronomy Bull 2017; 23: 46-48 (in China). https://doi. org/10.16377/j.cnki.issn1007-7731.2017.16.014

7. Kushimo, Omobolanle. The Tick Genus Amblyomma in Africa: Phylogeny and Mutilocus DNA Barcoding. Electronic Theses and Dissertations. 2013, p 835. https://digitalcommons.georgiasouthern.edu/etd/835

8. Sheng J, Jiang M, Yang M, Bo X, Zhao S, Zhang Y, Wureli H, Wang B, Tu C, Wang Y. Tick distribution in border Oblasts of Northwestern China. Ticks Tick Borne Dis 2017; 10: 665-669. https://doi.org/10.1016/j.ttbdis.2019.02.011

9. Nurmakhanov T, Sansyzbaev Y, Atshabar B, Deryabin P, Kazakov S, Zholshorinov A, Matzhanova A, Sadvakassova A, Saylaubekuly R, Kyraubaev K, Hay J, Atkinson B, Hewson R. Crimean-Congo haemorrhagic fever virus in Kazakhstan (1948-2013). Int J Infect Dis 2015; 38: 19-23. https://doi.org/10.1016/j.ijid.2015.07.007

10. Turebekov N, Abdiyeva K, Yegemberdiyeva R, Dmitrovsky A, Yeraliyeva L, Shapiyeva Z, Amirbekov A, Oradova A, Kachiyeva Z, Ziyadina L, Hoelscher M, Froeschl G, Dobler, G, Zinner J, Frey S, Essbauer S. Prevalence of Rickettsia species in ticks including identification of unknown species in two regions in Kazakhstan. Parasit Vectors. 2019; 12: 197. https://doi.org/10.1186/s13071019-3440-9

11. Folmer O, Black M, Hoeh W, Lutz R, Vrijenhoek R. DNA primers for amplification of mitochondrial cytochrome $c$ oxidase subunit I from diverse metazoan invertebrates. Mol Mar Biol Biotechnol 1994; 3: 294-299.

12. Tamura K, Nei M. Estimation of the number of nucleotide substitutions in the control region of mitochondrial DNA in humans and chimpanzees. Mol Biol Evol 1993; 10: 512-526. https://doi.org/10.1093/oxfordjournals.molbev.a040023

13. Librado P, Rozas J. DnaSP v5: a software for comprehensive analysis of DNA polymorphism data. Bioinformatics. 2009; 25: 1451-1452. https://doi.org/10.1093/bioinformatics/btp187

14. Bandelt HJ, Forster P, Röhl A. Median-joining networks for inferring intraspecific phylogenies. Mol Biol Evol 1999; 16: 37-48. https://doi.org/10.1093/oxfordjournals.molbev.a026036 
15. Wetjen M, Schmidt T, Schrimpf A, Schulz R. Genetic diversity and population structure of burbot Lota lota in Germany: Implications for conservation and management. Fish Manag Ecol 2020; 27: 170-184. https://doi.org/10.1111/fme.12396

16. Puffenberger EG, Hosoda K, Washington SS, Nakao K, deWit D, Yanagisawa M, Chakravart A. A missense mutation of the endothelin-B receptor gene in multigenic Hirschsprung's disease. Cell 1994; 79: 1257-1266. https://doi.org/10.1016/0092-8674(94)90016-7

17. Apanaskevich DA, Filippova NA, Horak IG. The genus Hyalomma Koch, 1844. X. Redescription of all parasitic stages of $H$. (Euhyalomma) scupense Schulze, 1919 (=H. detritum Schulze) (Acari: Ixodidae) and notes on its biology. Folia Parasitol (Praha) 2010; 57: 69-78. https://doi.org/10.14411/fp.2010.009

18. Chandra S, Ma GC, Burleigh A, Brown G, Norrisa JM, Ward MP,
Emerya D, Šlapetaa J. The brown dog tick Rhipicephalus sanguineus sensu Roberts, 1965 across Australia: Morphological and molecular identification of $R$. sanguineus s.l. tropical lineage. Ticks Tick Borne Dis 2020; 11: 101305. https://doi.org/10.1016/j.ttbdis. 2019.101305

19. Zemtsova GE, Apanaskevich DA, Reeves WK, Hahn M, Snellgrove A, Levin ML Phylogeography of Rhipicephalus sanguineus sensu lato and its relationships with climatic factors. Exp Appl Acarol 2016; 69: 191-203. https://doi.org/10.1007/s10493-0160035-4

20. Guo LP, Mu LM, Xu J, Jiang SH, Wang AD, Chen CF, Guo G, Zhang WJ, Wang YZ. Rickettsia raoultii in Haemaphysalis erinacei from marbled polecats, China-Kazakhstan border. Parasit Vectors 2015; 8: 461. https://doi.org/10.1186/s13071-015-1065-1 